\title{
Abandoning Prophylactic Abdominal Drainage after Hepatic Surgery: 10 Years of No-Drain Policy in an Enhanced Recovery after Surgery Environment
}

\author{
Edgar M. Wong-Lun-Hing ${ }^{a}$ Victor van Woerden ${ }^{a}$ Toine M. Lodewick ${ }^{a, b}$ \\ Marc H.A. Bemelmans ${ }^{a, b}$ Steven W.M. Olde Damink ${ }^{a-c}$ Cornelis H.C. Dejong $^{a-d}$ \\ Ronald M. van Dama, b \\ ${ }^{a}$ Department of Surgery, Maastricht University Medical Centre (MUMC), ${ }^{b}$ ESCAM (European Surgical Centre Aachen \\ Maastricht), ' Nutrim School for Nutrition and Translational Research in Metabolism, and ${ }^{\mathrm{d}}$ GROW, School for Oncology and \\ Developmental Biology, Maastricht, The Netherlands
}

\section{Keywords}

Drain · No-drain $\cdot$ Prophylactic drainage $\cdot$ Abdominal drainage $\cdot$ Liver surgery

\begin{abstract}
Background: Routine prophylactic abdominal drainage after hepatic surgery is still being debated, as it may be unnecessary, possibly harmful, and uncomfortable for patients. This study evaluated the safety of a no-drain policy after liver resection within an Enhanced Recovery after Surgery (ERAS) programme. Methods: All hepatectomies performed without prophylactic drainage during 2005-2014 were included. Primary end points were resection-surface-related (RSR) morbidity, defined as the presence of postoperative biloma, hemorrhage or abscess, and reinterventions. Secondary end points were length of stay, total postoperative morbidity, the composite end point of liver surgery-specific complications, readmissions, and 90-day mortality. Uniand multivariate analyses were performed to identify independent risk factors for RSR morbidity. A systematic search was performed to compare the results of this study to literature. Results: A total of 538 resections were included in the study. The RSR complication and reintervention rate was 15 and $12 \%$, respectively. Major liver resection ( $\geq 3$ segments)
\end{abstract}

\section{KARGER}

E-Mail karger@karger.com www.karger.com/dsu
This article is licensed under the Creative Commons AttributionNonCommercial-NoDerivatives 4.0 International License (CC BYNC-ND) (http://www.karger.com/Services/OpenAccessLicense). Usage and distribution for commercial purposes as well as any dis tribution of modified material requires written permission. was an independent risk factor for the development of RSR morbidity (OR 3.01, 95\% Cl 1.61-5.62; $p=0.001)$ and need for RSR reintervention (OR $3.02,95 \% \mathrm{Cl} 1.59-5.73 ; p=0.001)$. Conclusion: RSR morbidity, mortality, and reintervention rates after liver surgery without prophylactic drainage in patients, treated within an ERAS programme, were comparable to previously published data. A no-drain policy after partial hepatectomy seems safe and feasible.

(C) 2017 The Author(s)

Published by S. Karger AG, Basel

\section{Introduction}

In the past decades, the quality of perioperative care for hepatic surgery has improved dramatically due to improvements in surgical technique, risk assessment and perioperative care. Prophylactic intra-abdominal drainage is still routinely applied in many hospitals worldwide. However, prophylactic intra-abdominal drainage may be unnecessary [1], uncomfortable, and even harmful for the patient. As early as 1915, the British surgeon, Major Grey

E.M.W.-L.-H. and V.W. contributed equally to this work. This study has been presented with preliminary results as an oral presentation at the E-AHPBA 2013 congress in Belgrade, Serbia.

Ronald M. van Dam

Department of Surgery

Maastricht University Medical Centre

PO Box 5800, NL-6202 AZ Maastricht (The Netherlands)

E-Mail r.van.dam@mumc.nl 
Turner, already pointed out the dangers of routine drain use. One hundred years later, the routine use of prophylactic drains in liver surgery is still an on-going debate [2].

Some studies have reported advantages of prophylactic drain placement such as early drainage of bile leaks, preventing subphrenic collection, detecting postoperative hemorrhage, and removing ascites [3-6]. Other studies have indicated that the risks of prophylactic intra-abdominal drains, including drain-related bleeds, ascending intra-abdominal infections by retrograde contamination, and impaired pulmonary function, outweigh the benefits $[1,7,8]$. Furthermore, patients with a drain in place experience more abdominal pain and discomfort, have more difficulties to mobilize, need more nursing care, and this could contribute to a longer admission and increased costs [7].

Currently, if used, drains are usually placed near the transection surface or in the subphrenic space and are often removed on postoperative day 3-5 depending on drain production $[7,9,10]$. Already in 2007 , a meta-analysis [11] showed that there were no significant differences in morbidity, mortality, and re-operation rates between patients with or without a prophylactic abdominal drain after uncomplicated elective hepatectomy. Postoperative mortality in patients undergoing liver surgery without a drain ranges between 0 and $3 \%$. Percutaneous or operative reintervention rates in patients without a drain are $0-18 \%$ and $0-10 \%$, respectively $[1,4,7,8,12-14]$, and do not differ from outcomes after placement of an abdominal drain. Patients with a drain have reported mortality, percutaneous reintervention, and re-operation rates of $0-6,0-36$, and $0-6 \%$, respectively $[1,4,7,8,12-14]$. The accumulating evidence suggests that routine abdominal drainage after liver resection is unnecessary and the benefit arguable, but it may be indicated in specific patients.

The Enhanced Recovery after Surgery (ERAS) programme has shown a benefit for patient recovery after liver surgery in recent years [15-17]. The programme has been implemented to optimize pre-, peri-, and postoperative care to facilitate a quicker recovery. With the introduction of this programme, the routine use of drains after liver surgery without biliary or vascular reconstruction has been abandoned [15-17].

The aim of this study was to examine the postoperative complication and reintervention rates in patients undergoing liver resection that were treated without prophylactic drains in an ERAS environment, to identify risk factors associated with reinterventions for specific complications, and to compare the results to earlier studies on drainage following liver resection.

\section{Methods}

Study Design

All patients undergoing hepatic resection between January 2005 and December 2014 at Maastricht University Medical Centre (MUMC) were included in a prospective database. Patients were retrospectively identified and screened for eligibility in this study. Patients were excluded if a prophylactic abdominal drain was placed, for example, in the case of hepatic resection traumatic lesions, or a bilioenteric anastomosis was created. Primary end points of this study were resection-surface-related (RSR) morbidity and reinterventions for RSR morbidity. RSR morbidity was defined as the presence of bile leakage, intra-abdominal abscess, or/ and hemorrhage [18]. Reinterventions for RSR morbidity were considered to be CT- or US-guided percutaneous drainage, re-operation, and endoscopic retrograde cholangiopancreaticography with stenting. Data on reinterventions were retrospectively collected from individual patient charts. Secondary end-points were hospital length of stay (LOS), postoperative morbidity, readmission rate, 90-day mortality, and the composite end point of liver surgery-specific complications (CEP). A composite end point consists of 2 or more specific complications that can be regarded as 1 dichotomous end point that occur in 1 patient. The liver surgeryspecific CEP consists of ascites, postresectional liver failure, bile leakage, intra-abdominal hemorrhage, intra-abdominal abscess, and/or postoperative mortality [19].

Surgical Techniques

All hepatic resections were performed by 1 of 4 hepatobiliary surgeons (C.H.C.D., R.M.D., S.W.M.O.D., and M.H.B.) or by a senior resident/fellow under the supervision of a hepatobiliary surgeon. Hepatectomies were performed as open or laparoscopic procedures as published previously [20].

For open procedures, a unilateral right subcostal, a bilateral subcostal (right extended to left), J-shaped, or midline incision was used. Intraoperative ultrasound was routinely performed to examine the location of lesions in the liver and the relation to surrounding biliary and vascular structures. Hepatic resection was performed by using a Cavitron Ultrasonic Surgical Aspirator (CUSA; System 200 Macrodissector, Cavitron Surgical Systems, USA) and argon beam coagulation (Force GSU System, Valleylab, USA), with or without Pringle's maneuver, and Ultracision Harmonic ACE (Ethicon Endosurgery, Johnson \& Johnson, USA). Central venous pressure was maintained at $\leq 5 \mathrm{~cm} \mathrm{H}_{2} \mathrm{O}$ during transection to reduce excessive blood loss. During transection, clips and ligatures were used to treat vessels and bile ducts at the resection surface. To avoid postoperative hemorrhage and bile leakage, the resection surface was treated with argon beam coagulation and or sealants at the discretion of the surgeon, although the effectiveness has never been proven [18].

For laparoscopic procedures, patients were placed in supine French position. Access to the abdomen was created by an open transumbilical insertion of a $30^{\circ}$ laparoscope. Three or 4 additional trocars were inserted. The pressure of the pneumoperitoneum was kept at $<12 \mathrm{~mm} \mathrm{Hg}$. Parenchymal transection was performed using the LigaSure $5 \mathrm{~mm}$ blunt tip (Covidien, USA), laparoscopic CUSA (Cavitron Surgical Systems, USA), or Harmonic scalpel (Ultracision, Ethicon Endosurgery, Johnson \& Johnson, USA). The portal pedicles were stapled using a vascular stapler (Endo GIA Autosuture, Covidien, USA). Resected specimens were placed
412

Dig Surg 2017;34:411-420

DOI: $10.1159 / 000455246$
Wong-Lun-Hing et al. 
in a plastic bag (Endo Catch Autosuture, Covidien, USA) and removed through a separate usually suprapubic muscle sparing incision.

\section{No-Drain Policy}

Since the introduction of ERAS in liver surgery in 2005, the MUMC has a no-drain policy; abdominal drains are no longer part of standard management after hepatectomy. The use of prophylactic abdominal drains was limited to a selected group of indications. They were routinely placed after the creation of bilioenteric anastomoses or biliary reconstructions and in the case of traumatic liver lesions. In some cases, placement of a prophylactic abdominal drain could be considered by the surgeon when a high risk of intraabdominal fluid collection was expected, for example, when performing combined procedures, in the case of intraoperative iatrogenic laceration that required drainage, repeat hepatectomies, excessively large resection surfaces, or central liver resections. Furthermore, all patients were treated within the ERAS programme. Key principles of this programme have been previously described [21]. Patients were closely monitored during hospitalization based on clinical presentation, vital parameters, and standard diagnostic laboratory results. Additional imaging (CT or US) was only performed on clinical findings indicative of postoperative complications. All complications, defined as any deviation from the expected postoperative course $<30$ days and graded according to the Clavien-Dindo classification system [22], were recorded in the electronic patient record system and in a prospectively maintained research database. Data from the electronic patient record system and the research database were crosschecked for missing data.

\section{Systematic Literature Search}

A systematic search was conducted to compare the results of this study with literature following the current recommendations of Preferred Reporting Items for Systematic Reviews and Metaanalysis Approach (PRISMA). Two authors (E.M.W.-L.-H. and V.W.) independently performed the search, study selection, data extraction, and critical appraisal of the studies.

\section{Eligibility Criteria}

After review of the abstract, the remaining studies were selected for full text evaluation and inclusion under the following conditions: (1) the subject of the study was a comparison of the routine use of an abdominal drain vs. no-drainage after hepatectomy; (2) the study was not an editorial, systematic review, or meta-analysis; (3) the study compared clinical outcome after abdominal drain vs. no-drainage after hepatectomy; and (4) the study was in the English language.

\section{Study Selection and Quality Appraisal}

The search was conducted in Ovid MEDLINE, Embase, and PubMed databases to identify all studies comparing the routine use of an abdominal drain vs. no-drainage after hepatectomy between January 1, 1990, and December 31, 2014. The following search strategy was used: ([["Hepatectomy" [Mesh] OR "liver resection" OR "liver surgery"]] AND ["Drainage" [Mesh] OR "drain*"] NOT ["preoperative drainage" OR "preoperative biliary drainage"]). After removal of duplicates, articles were screened by title, abstract, and subsequently full text. In addition, reference lists of all included studies were screened for missed but relevant

Abandoning Prophylactic Abdominal Drainage after Hepatic Surgery studies. The methodological quality and risk of bias of the studies was assessed using the Cochrane Handbook for Systematic Reviews [23]. All included studies were consequently graded by using the Oxford Centre for Evidence-Based Medicine levels of evidence [24]. Furthermore, clinical trial registers were searched for ongoing studies.

\section{Data Collection}

Two reviewers (E.M.W.-L.-H. and V.W.) independently extracted data from the selected studies on study design, participant characteristics, mortality, image-guided drainage, re-operation, bile leakage/fistula, infected collections, postoperative bleeding, and wound infection.

\section{Statistical Analysis}

Continuous data are described as median (range) and categorical data are presented as percentages. The chi-square/Fisher's exact and Mann-Whitney U tests were used to compare categorical data and continuous data, respectively. Results were considered significant when $p \leq 0.05$. Uni- and multivariate analyses were performed to define specific independent risk factors for RSR reinterventions. Variables included in the univariate analysis: sex, ASA class $\geq$ III, age $<65$ or $\geq 70$ years, median BMI, type of liver resection (major, caudate, repeat, central), blood loss $>2,000 \mathrm{~mL}$, preoperative chemotherapy, Pringle maneuver, and operating time $>240$ min. Multivariate analysis was performed with binary logistic regression, and $p \leq 0.10$ was used to select variables from univariate analysis. All statistical analyses were performed using IBM SPSS Statistics for Windows, version 20.0 (IBM Corp., Armonk, NY, USA).

\section{Results}

\section{General and Surgical Characteristics}

A total of 606 hepatic resections were performed in the study period. All the 66 patients who received a drain were excluded from further analysis. Details of this drain group will be addressed later in this section. Furthermore, 2 patients with missing data were excluded from analysis. A total of 538 patients were analyzed. General and surgical characteristics are shown in Table 1.

\section{Primary Outcome}

Seventy-nine (15\%) of the 538 patients without a drain developed postoperative RSR complications. Sixty-seven (12\%) of these patients required surgical, radiological, or endoscopic reintervention (Table 2).

Of the variables included in the univariate analysis (Tables 3,4 ), only age $<65$ years, major liver resection, blood loss $>2,000 \mathrm{~mL}$, Pringle maneuver, operating time $>240 \mathrm{~min}$, and preoperative chemotherapy were significantly $(p<0.1)$ associated with the development major RSR morbidity (Clavien-Dindo grade $\geq 3 \mathrm{a}$ ) or the need for postoperative RSR reinterventions.

Dig Surg 2017;34:411-420

DOI: $10.1159 / 000455246$ 
Table 1. Baseline patient and surgical characteristics

\begin{tabular}{|c|c|}
\hline & $\begin{array}{l}\text { All patients } \\
(n=538), \%\end{array}$ \\
\hline Gender, male & $308(57)$ \\
\hline Age, years & $64(55-70)$ \\
\hline $\mathrm{BMI}, \mathrm{kg} / \mathrm{m}^{2}$ & $25.6(22.9-28.5)$ \\
\hline \multicolumn{2}{|l|}{ ASA physical status } \\
\hline I & $85(16)$ \\
\hline II & $339(63)$ \\
\hline$\geq$ III & $114(21)$ \\
\hline \multicolumn{2}{|l|}{ Indications } \\
\hline Colorectal metastasis & $421(78)$ \\
\hline Benign lesions & $47(9)$ \\
\hline Other malignancy & $29(5)$ \\
\hline $\mathrm{HCC}$ & $24(5)$ \\
\hline Gall bladder carcinoma & $7(1)$ \\
\hline CCC & $5(1)$ \\
\hline Other & $5(1)$ \\
\hline Preoperative chemotherapy & $271(50)$ \\
\hline \multicolumn{2}{|l|}{ Resection type } \\
\hline$<1$ segment/metastasectomy & $213(40)$ \\
\hline Multisegmentectomy & $173(32)$ \\
\hline Right hemihepatectomy & $74(14)$ \\
\hline Right hemihepatectomy +1 segment & $31(6)$ \\
\hline Left hemihepatectomy & $16(3)$ \\
\hline Left hemihepatectomy +1 segment & $2(0.4)$ \\
\hline Right extended hemihepatectomy & $12(2)$ \\
\hline Left extended hemihepatectomy & $3(0.6)$ \\
\hline Central resection & $18(3)$ \\
\hline Caudate lobe resection & $25(5)$ \\
\hline Major hepatectomy ( $\geq 3$ segments) & $226(42)$ \\
\hline Repeat hepatectomy & $57(11)$ \\
\hline Total operating time, $\min$ & $200(150-270)$ \\
\hline Intraoperative blood loss, $\mathrm{mL}$ & $500(269-1,000)$ \\
\hline Pringle & $118(22)$ \\
\hline
\end{tabular}

Values in parentheses are percentages, unless indicated otherwise. Numeric data are presented as median (interquartile range). BMI, body mass index; ASA, American Society of Anesthesiologists; HCC, hepatocellular carcinoma; CCC, cholangiocellular carcinoma.

After multivariate analysis, major liver resection was an independent risk factor (OR 3.01, 95\% CI 1.615.62; $p=0.001$ ) for the development of major RSR morbidity and also associated with an increased risk of RSR reinterventions (OR 3.02, 95\% CI 1.59-5.73; $p=$ $0.001)$.

\section{Secondary Outcome Measures}

The median LOS was 8 days (6-11). Fifty patients (9\%) were readmitted within 30 days after surgery (Table 5). Mortality within 90 days after surgery was observed in 15 patients (2.8\%). Within this group, 5 patients died from sepsis following an intra-abdominal abscess or biloma,
Table 2. Primary end points

\begin{tabular}{lc}
\hline & $\begin{array}{c}\text { All patients } \\
(n=538), \%\end{array}$ \\
\hline RSR complication rate & $79(15)$ \\
All surgery-related complication rate & $90(20)$ \\
Bile leakage (RSR) & $35(7)$ \\
Hemorrhage (RSR) & $9(2)$ \\
Intra-abdominal abscess (RSR) & $44(8)$ \\
Ascites & $12(2)$ \\
Postoperative liver failure & $17(3)$ \\
Pleural effusion & $18(3)$ \\
Sepsis & $15(3)$ \\
Wound infection & $28(5)$ \\
Reintervention rate for RSR complications & $67(12)$ \\
Reintervention rate & $72(13)$ \\
CT drainage & $55(10)$ \\
ERCP with stenting & $9(2)$ \\
US drainage & $8(2)$ \\
Relaparotomy & $11(2)$ \\
Relaparoscopy & $1(0.2)$ \\
Thoracotomy & $1(0.2)$ \\
Thoracic drainage & $8(2)$ \\
\hline
\end{tabular}

Values in parentheses are percentages. Multiple complications or reinterventions per patient were possible. A total of 178 surgeryrelated complications occurred in 90 patients. A total of 93 reinterventions occurred in 72 patients.

RSR, resection surface-related; ERCP, endoscopic retrograde cholangiopancreaticography; US, ultrasound.

3 patients died from multiorgan failure induced by postresectional liver failure, 3 patients died from extensive extra-hepatic metastatic disease and subsequent multiorgan failure, and 4 patients died of an acute cardiac arrest of which one was proven in autopsy to be caused by myocardial infarction.

\section{Prophylactic Abdominal Drain Group}

Sixty-six patients received a prophylactic abdominal drain and were excluded from this study. Among them were 25 patients who required a drain after a bilioenteric anastomosis, or another form of biliary reconstruction was created. One patient received a drain after liver surgery for a traumatic lesion. Eight patients obtained a drain because of a difficult repeat hepatectomy. Seven patients received a drain for combined procedures (4 colorectal resections, 2 pancreatic resections, and 1 kidney resection). In 5 patients, a drain was placed after intraoperative iatrogenic lesions of the bile duct, pancreas, or spleen. Central resections and large resection surfaces after surgery were indications for drain placement in 9 patients. Vascular involvement required drain place- 

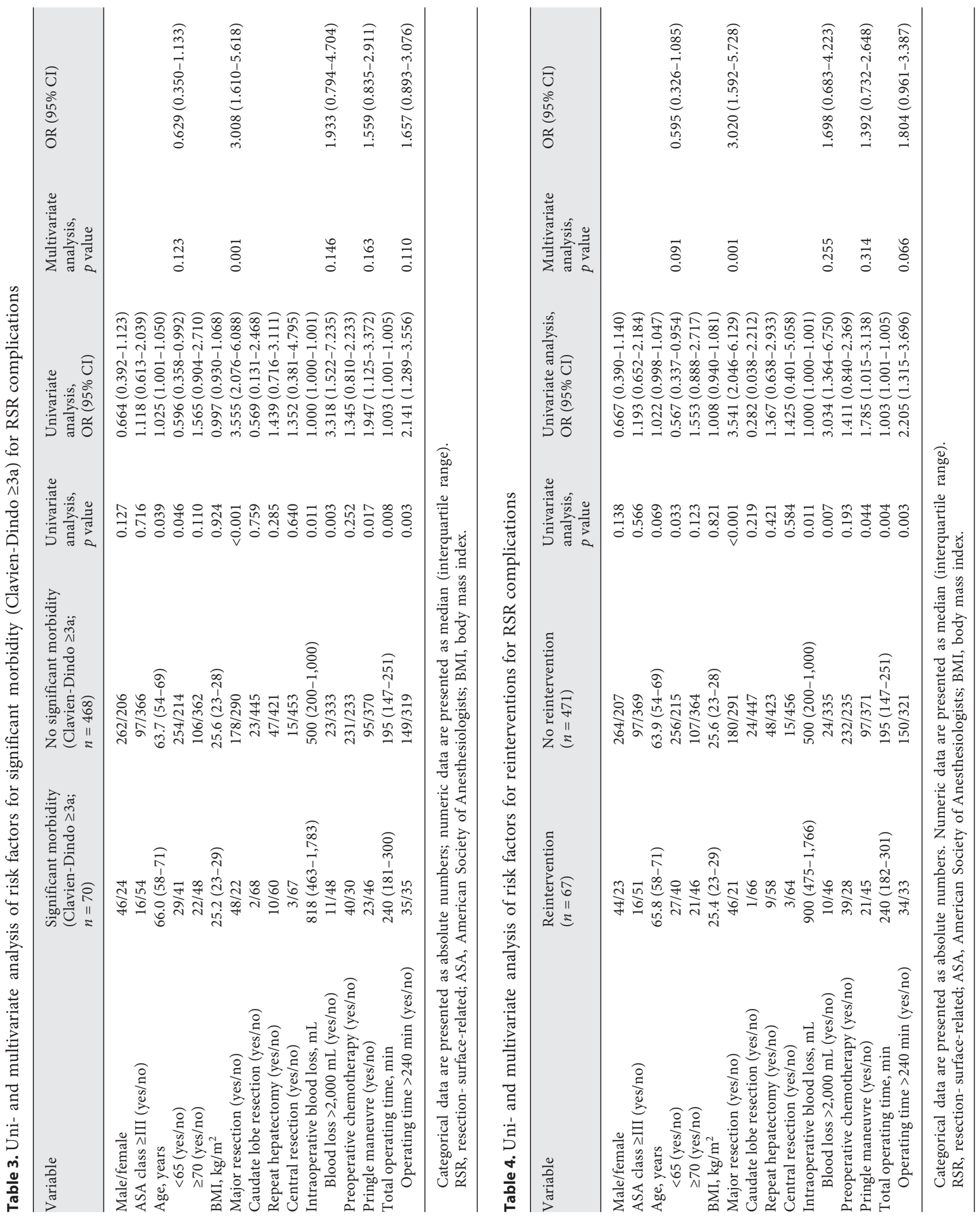
Fig. 1. PRISMA flow diagram of the literature search and inclusion of relevant studies.

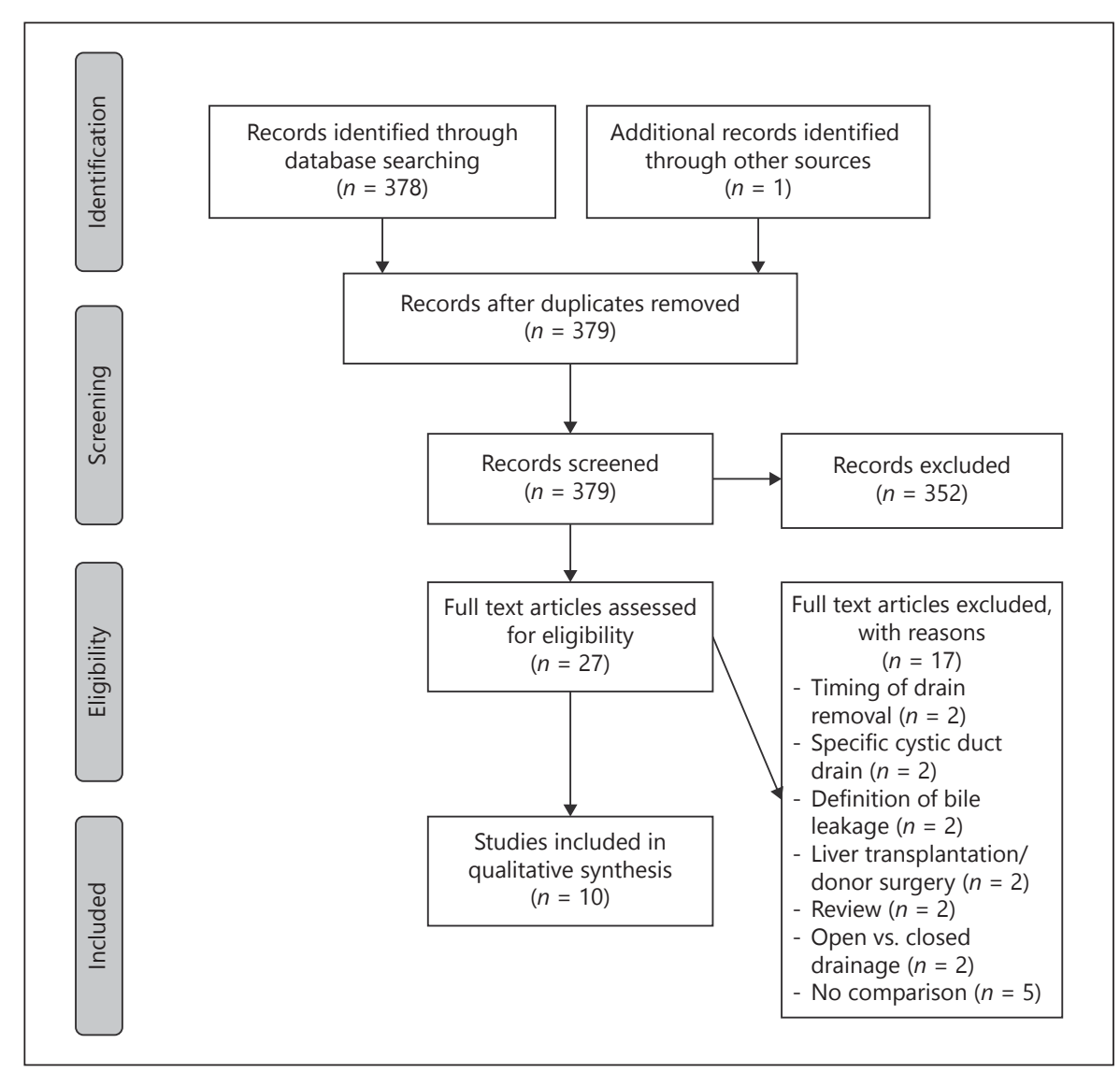

Table 5. Secondary outcomes

\begin{tabular}{lc}
\hline & $\begin{array}{c}\text { All patients } \\
(n=538), \%\end{array}$ \\
\hline $\begin{array}{l}\text { Readmission rate } \\
\text { Length of stay, days }\end{array}$ & $50(9)$ \\
Clavien-Dindo classification & $8(6-11)$ \\
$\quad$ Grade 1 & $33(6)$ \\
$\quad$ Grade 2 & $71(13)$ \\
$\quad$ Grade 3a & $55(10)$ \\
$\quad$ Grade 3b & $12(2)$ \\
Grade 5 (30-day mortality) & $14(3)$ \\
All reported postoperative morbidity & $13(2)$ \\
Major morbidity, Clavien-Dindo $\geq 3 \mathrm{a}$ & $202(38)$ \\
Major RSR morbidity, Clavien-Dindo $\geq 3 \mathrm{a}$ & $92(17)$ \\
Liver surgery-specific CEP & $70(13)$ \\
90-Day mortality & $84(16)$ \\
Liver-related 90-day mortality & $15(2.8)$ \\
\hline
\end{tabular}

Values in parentheses are percentages, unless indicated otherwise. Numeric data are presented as median (interquartile range).

RSR, resection- surface-related; CEP, liver surgery-specific composite end point (ascites, postresectional liver failure, bile leakage, intra-abdominal hemorrhage, intra-abdominal abscess, and operative mortality). ment in 2 patients. Two patients with liver cirrhosis received a prophylactic drain after surgery because of an expected high risk of ascites and subsequent infection. Another 2 patients received a drain in early 2005 because of protocol deviation by the surgeon. Lastly, in 5 patients, an abdominal drain was placed without any specified indication. In this selected drain group, excluded from analysis, morbidity and mortality were high (10.0\% mortality, 30.0\% RSR morbidity, 37.5\% major morbidity, $27.5 \%$ reinterventions).

\section{Literature Review}

The PRISMA flow diagram of the literature search and inclusion of relevant studies are shown in Figure 1. The search resulted in 27 studies that were assessed for eligibility. Finally, 10 studies, including 5 RCTs and 5 cohort studies ( 2 prospective and 3 retrospective series), were used to compare the results with those of the present study. The majority of studies included all types of liver resection, except 2 RCTs that only included patients with an underlying liver disease or a hepatocellular carcinoma in cirrhotic livers. Four studies $[1,4,7,8]$ were graded as 
level $1 \mathrm{~b}$ evidence, 1 study [13] was grade level $2 \mathrm{~b}$ evidence, and the remaining studies $[5,12,25-27]$ were cohort studies of grade 4 evidence. No ongoing studies could be identified in clinical trial registers. Table 6 shows a comparison of this study with results from literature.

\section{Discussion}

The use of postoperative drains after liver resection is still subject to debate. This study evaluated a no-drain policy, as part of the ERAS programme, in a tertiary referral center during 2005-2014. Implementation of a nodrain policy has resulted in an overall surgical morbidity rate of $20 \%$, RSR complication rate of $15 \%$, and RSR reintervention rate of $12 \%$.

This implies that $88 \%$ of all patients did not require any form of abdominal drainage in the postoperative phase. By not placing a prophylactic drain, they were spared from possible discomfort and harmful drain-related complications. Moreover, in the group of patients that did have an intra-abdominal collection, the majority of them could be treated well with CT- or US-guided drainage and rarely a reoperation was necessary, as is shown in Table 2.

Comparison of results of this study with earlier publications, mainly summarized by Gurusamy et al. [11], confirms the safety and feasibility of the no-drain policy. The 90 -day mortality of $2.8 \%$ in this study was within the ranges reported in the literature for this type of liver surgery $[1,4,7,8,11,13,14]$. Reintervention rates of previous studies $[1,4,7,8,11,13,14]$ vary considerably in studies of both drain and no-drain groups. To date, there is no evidence that abandoning prophylactic drainage increases the need for (radiological) reinterventions [11]. The RSR reintervention rate of $12 \%$ in this study demonstrates that a no-drain policy does not lead to more reinterventions, higher morbidity, or mortality compared to patients with a drain $[1,4,5,7,8,12,13,27]$.

Major liver resection is a known risk factor for postoperative complications [12, 13, 28-35]. In this study, it was identified in multivariate analysis as an independent risk factor for RSR-significant morbidity and RSR reinterventions. Other known intraoperative predictive determinants, such as prolonged operating time $[12,13,28,31,33$, 35], repeat hepatectomy [35-37], and the use of Pringle maneuver [31, 38], were not confirmed in this cohort. Preoperative risk factors that have been suggested in preceding literature, among which are the presence of significant comorbidities/ASA III-IV [28-30, 32, 39], an abnormal liver function $[13,28,30]$, and chemotherapy [40],

Abandoning Prophylactic Abdominal Drainage after Hepatic Surgery were also not confirmed. The fact that major liver resection was found as a risk factor does not mean that a prophylactic drain needs to be placed routinely. It implies that there is a subset of patients in which routine postoperative imaging or an aggressive postoperative imaging strategy needs to be considered. When there is a clinically relevant intra-abdominal collection on ultrasound or CT, a therapeutic radiologically guided drain can then be placed.

The expertise with, and access to, radiological reinterventions may vary substantially between institutions; it has changed over time in hospitals worldwide and it could be an important factor. In centers in which radiological reinterventions are less frequently performed, more collections may be inadequately drained. This could lead to a higher re-operation rate. When compared with patients receiving a drain in other studies, the patients from the present study had a low re-operation rate. This may imply that a no-drain placement policy does not lead to more re-operations. Finally, hospital LOS in other studies was longer [41]. The ERAS programme could explain this faster recovery [21, 42].

This study has several strengths that add to the existing body of evidence of RCTs and a meta-analysis on the studied topic. An important strength of this study is the large cohort size. A total of 538 consecutive patients were treated without a drain after implementation of the nodrain policy. As is demonstrated in Table 6, this no-drain cohort is one of the largest cohorts on the subject. In addition, the group of excluded patients with prophylactic drain placement is well described and provides detailed insight in the selection process. Furthermore, patients with bilioenteric anastomoses were excluded from analysis to enable comparison with previously published studies $[7,8,13]$. Among these patients, leakage rates are high and may confound general results. Another strength of this study is the fact that uni- and multivariate analyses were performed for the identification of risk factors for surface-related morbidity. This can aid in the decisionmaking process of abandoning the use of drains after liver surgery. Lastly, all patients were prospectively registered and treated within an ERAS programme consisting of standardized care elements. The minimal use of prophylactic drains is an important element in the ERAS programme, and this study advocates a no-drain policy after uncomplicated liver surgery. Drains are thought to affect postoperative mobility and pain control and drains could hamper a swift recovery in uncomplicated cases.

The retrospective analysis of prospectively collected data is a limitation of this study. Although all liver resections in the study period were registered, the final results

Dig Surg 2017;34:411-420

DOI: $10.1159 / 000455246$ 


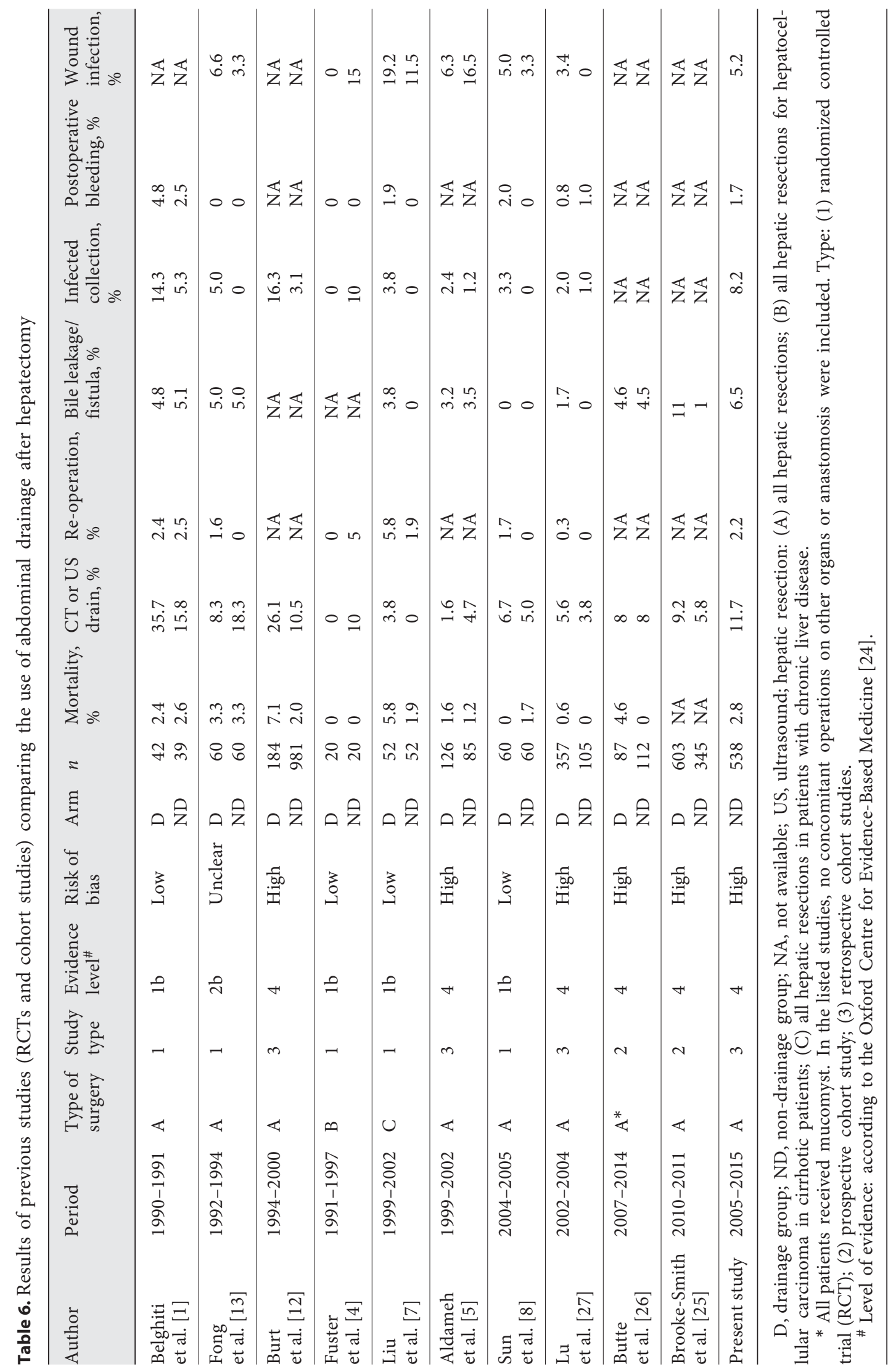


may have been prone to a form of selection bias, because a small subset of patients was excluded from analysis. This excluded group received an abdominal drain by discretion of the operating surgeon within the studied period. Although no biliary reconstructions were performed in these patients, drains were placed because of major combined procedures, major central liver resections, intraoperative leaks of the bile duct, pancreatic damage, and repeat hepatectomy.

The findings of this cohort study confirm the findings of available RCTs $[4,7,13]$ and a Cochrane review [11] that routine drainage after uncomplicated liver resection is not necessary. The results of this study show that a no-drain policy is safe and feasible after liver surgery within an ERAS environment. Placement of a prophylactic drain is unlikely to prevent reinterventions for complications and a no-drain policy seems justified in the majority of patients. At most, placement of a drain allows liver surgeons to detect complications in an earlier phase, but routine placement subjects a large group of patients to potential risks and discomfort. Further studies are still necessary and should focus on specific patient groups with predefined risk factors (e.g., underlying liver disease, major resections, biliary reconstructions, intraoperative blood loss, and operating time) or should validate risk factors.

\section{Conclusion}

A selective no-drain policy within an ERAS environment resulted in a rate of postoperative complications, reinterventions, and mortality that is comparable to previously published studies. The routine use of prophylactic abdominal drains after liver surgery therefore seems unnecessary. In patients that undergo a major liver resection, which is an independent risk factor for RSR-related complications, preemptive postoperative imaging can be considered.

In a small group of selected patients known to have a high risk of anastomotic leakage, prophylactic drains still have their place, for example, in the case of biliary reconstruction.

\section{Statement of Ethics}

The study was performed in line with GCP guidelines. Due to the retrospective nature of the study, informed consent of the study subjects was not required.

\section{Disclosure Statement}

The authors have no conflicts of interest or financial support to disclose.

\section{References}

1 Belghiti J, et al: Drainage after elective hepatic resection. A randomized trial. Ann Surg 1993; 218:748-753.

2 Turner MGG: A note on the abuses and dangers of drainage tubes: with special reference to the danger of serious hæmorrhage from the erosion of large arteries. Br J Surg 1915;3:552557.

3 Bona S, Gavelli A, Huguet C: The role of abdominal drainage after major hepatic resection. Am J Surg 1994;167:593-595.

4 Fuster J, et al: Abdominal drainage after liver resection for hepatocellular carcinoma in cirrhotic patients: a randomized controlled study. Hepatogastroenterology 2004;51:536-540.

5 Aldameh A, McCall JL, Koea JB: Is routine placement of surgical drains necessary after elective hepatectomy? Results from a single institution. J Gastrointest Surg 2005;9:667-671.

6 Petrowsky H, et al: Evidence-based value of prophylactic drainage in gastrointestinal surgery: a systematic review and meta-analyses. Ann Surg 2004;240:1074-1084; discussion 1084-1085.

7 Liu CL, et al: Abdominal drainage after hepatic resection is contraindicated in patients with chronic liver diseases. Ann Surg 2004; 239: 194-201.

Abandoning Prophylactic Abdominal Drainage after Hepatic Surgery
8 Sun HC, et al: Randomized clinical trial of the effects of abdominal drainage after elective hepatectomy using the crushing clamp method. Br J Surg 2006;93:422-426.

9 Tanaka K, et al: The effectiveness and appropriate management of abdominal drains in patients undergoing elective liver resection: a retrospective analysis and prospective case series. Surg Today 2013;43:372380.

10 Yamazaki S, et al: Criteria for drain removal following liver resection. Br J Surg 2012;99: 1584-1590.

11 Gurusamy KS, Samraj K, Davidson BR: Routine abdominal drainage for uncomplicated liver resection. Cochrane Database Syst Rev 2007;3:CD006232.

12 Burt BM, et al: An audit of results of a nodrainage practice policy after hepatectomy. Am J Surg 2002;184:441-445.

13 Fong Y, et al: Drainage is unnecessary after elective liver resection. Am J Surg 1996;171: 158-162.

14 Kyoden Y, et al: Value of prophylactic abdominal drainage in 1269 consecutive cases of elective liver resection. J Hepatobiliary Pancreat Sci 2010;17:186-192.
15 Coolsen MM, et al: A systematic review of outcomes in patients undergoing liver surgery in an enhanced recovery after surgery pathways. HPB (Oxford) 2013;15:245-251.

16 Hughes MJ, McNally S, Wigmore SJ: Enhanced recovery following liver surgery: a systematic review and meta-analysis. HPB (Oxford) 2014;16:699-706.

17 Jones $C$, et al: Randomized clinical trial on enhanced recovery versus standard care following open liver resection. Br J Surg 2013;100: 1015-1024.

18 de Boer MT, et al: Fibrin sealant for prevention of resection surface-related complications after liver resection: a randomized controlled trial. Ann Surg 2012;256:229-234.

19 van den Broek MA, et al: Development of a composite endpoint for randomized controlled trials in liver surgery. Br J Surg 2011; 98:1138-1145.

20 Dejong C, Garden O: Neoplasms of the liver, ed 1; in Majid A, Kingsnorth A (eds): Advanced Surgical Practice. London, Greenwich Medical Media, 2003.

21 van Dam RM, et al: Initial experience with a multimodal enhanced recovery programme in patients undergoing liver resection. $\mathrm{Br} \mathrm{J}$ Surg 2008;95:969-975. 
22 Dindo D, Demartines N, Clavien PA: Classification of surgical complications: a new proposal with evaluation in a cohort of $6336 \mathrm{pa}-$ tients and results of a survey. Ann Surg 2004; 240:205-213.

23 Higgins J, Green S: Cochrane Handbook for Systematic Reviews of Interventions Version 5.1.0. The Cochrane Collaboration. 2011. http://www.cochrane-handbook.org/ (June 26, 2016).

24 Phillips R, et al: Oxford Centre for Evidencebased Medicine - Levels of Evidence, March 2009. http://www.cebm.net/oxford-centreevidence-based-medicine-levels-evidencemarch-2009 (June 26, 2016).

25 Brooke-Smith M, et al: Prospective evaluation of the international study group for liver surgery definition of bile leak after a liver resection and the role of routine operative drainage: an international multicentre study. HPB (Oxford) 2015;17:46-51.

26 Butte JM, et al: The role of peri-hepatic drain placement in liver surgery: a prospective analysis. HPB (Oxford) 2014;16:936-942.

27 Lu L, et al: Abdominal drainage was unnecessary after hepatectomy using the conventional clamp crushing technique. J Gastrointest Surg 2006;10:302-308.
28 Aloia TA, et al: Predicting poor outcome following hepatectomy: analysis of 2313 hepatectomies in the NSQIP database. HPB (Oxford) 2009;11:510-515.

29 Andres A, et al: Complications of elective liver resections in a center with low mortality: a simple score to predict morbidity. Arch Surg 2011;146:1246-1252.

30 Breitenstein S, et al: Novel and simple preoperative score predicting complications after liver resection in noncirrhotic patients. Ann Surg 2010;252:726-734.

31 Erdogan D, et al: Morbidity and mortality after liver resection for benign and malignant hepatobiliary lesions. Liver Int 2009;29:175180.

32 Farges O, et al: Incidence and risks of liver resection: an all-inclusive French nationwide study. Ann Surg 2012;256:697-704; discussion 704-705.

33 Imamura $\mathrm{H}$, et al: One thousand fifty-six hepatectomies without mortality in 8 years. Arch Surg 2003;138:1198-1206; discussion 1206.

34 Jarnagin WR, et al: Improvement in perioperative outcome after hepatic resection: analysis of 1,803 consecutive cases over the past decade. Ann Surg 2002;236:397-406; discussion 406-407.

35 Sadamori H, et al: Intractable bile leakage after hepatectomy for hepatocellular carcinoma in 359 recent cases. Dig Surg 2012;29:149-156.
36 Hirokawa F, et al: Re-evaluation of the necessity of prophylactic drainage after liver resection. Am Surg 2011;77:539-544.

37 Zimmitti G, et al: Greater complexity of liver surgery is not associated with an increased incidence of liver-related complications except for bile leak: an experience with 2,628 consecutive resections. J Gastrointest Surg 2013;17:57-64; discussion 6465.

38 Benzoni E, et al: Liver resective surgery: a multivariate analysis of postoperative outcome and complication. Langenbecks Arch Surg 2007;392:45-54

39 Schroeder RA, et al: Predictive indices of morbidity and mortality after liver resection. Ann Surg 2006;243:373-379.

40 Ishizawa T, et al: Using a 'no drain' policy in 342 laparoscopic hepatectomies: which factors predict failure? HPB (Oxford) 2014; 16: 494-499.

41 Gurusamy KS, Samraj K, Davidson BR: Routine abdominal drainage for uncomplicated liver resection. Cochrane Database Syst Rev 2007;3:CD006232.

42 Schultz NA, et al: Evaluation of a fast-track programme for patients undergoing liver resection. Br J Surg 2013;100:138-143. 